\title{
ANNALS OF ONCOLOGY
}

An official Journal of the European Society for Medical Oncology and the official journal of the Japanese Society of Medical Oncology

\section{TABLE OF CONTENTS}

$$
\bullet
$$$$
\text { Description }
$$$$
\text { - Impact Factor }
$$$$
\text { - } \quad \text { Abstracting and Indexing }
$$$$
\text { - Editorial Board }
$$$$
\text { - Guide for Authors }
$$
p.1
p.1
p.1
p.2
p.5

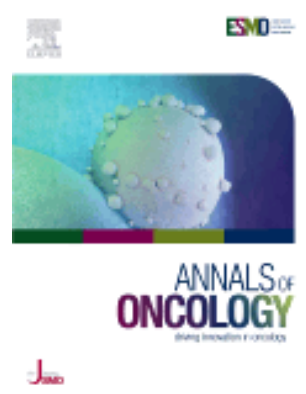

ISSN: 0923-7534

\section{DESCRIPTION}

Annals of Oncology, the journal of the European Society for Medical Oncology and the Japanese Society of Medical Oncology, provides rapid and efficient peer-review publications on innovative cancer treatments or translational work related to oncology and precision medicine.

Main focuses of interest include: systemic anticancer therapy (with specific interest on molecular targeted agents and new immune therapies), randomized trials (including negatives ones), top-level guidelines, and new fields currently emerging as key components of personalized medicine, such as molecular pathology, bioinformatics, modern statistics, and biotechnologies. Radiotherapy, surgery and pediatrics manuscripts can be considered if they display a clear interaction with one of the fields above or are paradigm-shifting.

With a large international editorial board of experts who are leaders in their fields, Annals of Oncology aims at delivering the best communication on the fast moving, and continually evolving, global oncology landscape.

\section{IMPACT FACTOR}

2021: 51.769 @ Clarivate Analytics Journal Citation Reports 2022

\section{ABSTRACTING AND INDEXING}

Current Contents - Clinical Medicine Elsevier BIOBASE

Current Awareness in Biological Sciences

Embase

Journal Citation Reports - Science Edition

ProQuest

PubMed Central

Science Citation Index Expanded

Science Citation Index 


\section{EDITORIAL BOARD}

\section{Editor-in-Chief}

Fabrice André, Villejuif, France

Deputy Editor

Daniel George Haller, Philadelphia, Pennsylvania, United States of America

Associate Editors

Urogenital tumors

Gerhardt Attard, London, United Kingdom

\section{Gastrointestinal tumors}

Dirk Arnold, Hamburg, Germany

Andrés Cervantes, Valencia, Spain

Josep Tabernero, Barcelona, Spain

\section{Breast tumors}

Christos Sotiriou, Brussels, Belgium

Thoracic tumors

Solange Peters, Lausanne, Switzerland

Johan F. Vansteenkiste, Leuven, Belgium

Head and neck tumors

Anthony T. C. Chan, Hong Kong, China

Gynecological tumors

Bradley J. Monk, Phoenix, Arizona, United States of America

Sandro Pignata, Napoli, Italy

Melanoma

Georgina V. Long, North Sydney, Australia

Hematological malignancies

Kunihiro Tsukasaki, Hidaka, Japan

Supportive care

Karin Jordan, Potsdam, Germany

\section{Epidemiology}

Paolo Boffetta, New York, New York, United States of America

Pagona Lagiou, Athens, Greece

Preclinical and experimental science

Thomas U. E. Helleday, Sheffield, United Kingdom

Precision medicine

Charles Swanton, London, United Kingdom

Bioinformatics

Nicholas McGranahan, London, United Kingdom

\section{Onco-Immunology}

Paolo A. Ascierto, Napoli, Italy

Molecular and surgical pathology

Ignacio Wistuba, Houston, Texas, United States of America

Early drug development

Christophe Massard, Villejuif, France

Liaison with ESMO

Pilar Garrido, Madrid, Spain

Industry corner: perspectives and controversies

Kapil Dhingra, Sparta, Tennessee, United States of America 


\section{Methodology}

Lisa Belin, Paris, France

Diana Giannarelli, Roma, Italy

Axel Hinke, Düsseldorf, Germany

Víctor Moreno, L'Hospitalet de Llobregat, Spain

Social media

Philippe Aftimos, Brussels, Belgium

Biotechnologies

Seiji Yano, Kanazawa, Japan

\section{Editorial Board}

Matti S. Aapro, Genolier, Switzerland

Maria Alsina, Barcelona, Spain

Yuichi Ando, Nagoya, Japan

Isobel Barnes, Oxford, United Kingdom

Joaquim Bellmunt, Boston, Massachusetts, United States of America

Benjamin Besse, Villejuif, France

Joerg Beyer, Zurich, Switzerland

Jean-Yves Blay, Villeurbanne, France

Carsten Bokemeyer, Hamburg, Germany

Emilio Bria, Roma, Italy

Earle F. Burgess, Charlotte, North Carolina, United States of America

Paolo Casali, Milano, Italy

Fortunato Ciardiello, Caserta, Italy

Alessandro Comandone, Torino, Italy

Philippa Corrie, Cambridge, United Kingdom

Javier Cortes, Barcelona, Spain

Giuseppe Curigliano, Milano, Italy

Rodrigo Dienstmann, Barcelona, Spain

Tanya Dorff, Duarte, California, United States of America

Alexandru Eniu, Cluj-Napoca, Romania

Boon-Cher Goh, Singapore, Singapore

Peter S. Grimison, Camperdown, New South Wales, Australia

Susan Halabi, Durham, North Carolina, United States of America

David M. Hyman, New York, New York, United States of America

Michail Ignatiadis, Bruxelles, Belgium

David H. Ilson, New York, New York, United States of America

Akira Inoue, Sendai, Japan

Chikashi Ishioka, Sendai, Japan

Hiroji Iwata, Aichi, Japan

Filip Janku, Houston, Texas, United States of America

Noriyuki Katsumata, Bunkyo-Ku, Japan

Tomoya Kawaguchi, Osaka, Japan

Naomi Kiyota, Kobe, Japan

Carlo La Vecchia, Milano, Italy

Primo N. Lara Jr., Sacramento, California, United States of America

James M. Larkin, London, United Kingdom

Sherene Loi, Melbourne, Australia

Sibylle Loibl, Neu-Isenburg, Germany

Florian Lordick, Leipzig, Germany

Yohann Loriot, Villejuif, France

Domenica (Ketta) Lorusso, Milano, Italy

Teresa Macarulla Mercade, Barcelona, Spain

Miguel Martín Jiménez, Madrid, Spain

Shigeyuki Matsui, Nagoya, Japan

Joan Maurel, Barcelona, Spain

Grant McArthur, Melbourne, Victoria, Australia

Funda Meric-Bernstam, Houston, Texas, United States of America

Stefan Michiels, Villejuif, France

Hironobu Minami, Kobe, Japan

Tony Mok, Hong Kong, Hong Kong

Manabu Muto, Kyoto, Japan

Yasumasa Nishimura, Osakasayama, Japan

Kazuto Nishio, Osaka-Sayama, Japan

Silvia Novello, Torino, Italy 
Michinori Ogura, Kakamigahara, Japan

Atsushi Ohtsu, Kashiwa, Japan

Isamu Okamoto, Fukuoka, Japan

Sai-Hong Ignatius Ou, Irvine, California, United States of America

Carmel Pezaro, Box Hill, Australia

Per Pfeiffer, Odense, Denmark

Sophie Postel-Vinay, Villejuif, France

Amanda Psyrri, Athens, Greece

Suresh Ramalingam, Atlanta, Georgia, United States of America

Martin Reck, Großhansdorf, Germany

Brian I. Rini, Cleveland, Ohio, United States of America

Rafael Rosell, Badalona, Spain

Shigehira Saji, Kyoto, Japan

Ramon Salazar, Barcelona, Spain

Taroh Satoh, Osakasayama, Japan

Mario Scartozzi, Ancona, Italy

Norbert Schmitz, Münster, Germany

Hans-Joachim Schmoll, Halle, Germany

Qian Shi, Rochester, Minnesota, United States of America

Alberto Sobrero, Genova, Italy

Guru Sonpavde, Houston, Texas, United States of America

Vivek Subbiah, Houston, Texas, United States of America

Shunji Takahashi, Tokyo, Japan

Nick Turner, London, United Kingdom

Eric Van Cutsem, Leuven, Belgium

Yi-Long Wu, Guangzhou, China

James Chih-Hsin Yang, Taipei, Taiwan

Takayuki Yoshino, Kashiwa, Japan

Andrew Zhu, Boston, Massachusetts, United States of America

Editors Emeriti

Franco Cavalli, Bellinzona, Switzerland

David Kerr, Oxford, United Kingdom

Jean-Charles Soria, Villejuif, France

Jan B. Vermorken, Edegem, Belgium 


\title{
GUIDE FOR AUTHORS
}

\section{INTRODUCTION}

Guide for Authors updated 29th November 2022

\section{Annals of Oncology - Editorial Office}

\author{
Annals of Oncology \\ Via Ginevra 4 \\ $\mathrm{CH}-6900$ Lugano \\ Switzerland
}

Telephone: $+41(0) 91-973-1908 / 10$

\section{E-mail: Annals1@esmo.org/Annals2@esmo.org}

Annals of Oncology publishes manuscripts that describe new findings of particular significance in any area related to clinical oncology and clinically-oriented basic cancer research. The criteria for acceptance are originality and high scientific quality. Manuscripts should be submitted with a letter specifying that the report is not under consideration for publication elsewhere and that all named authors have agreed to its submission. Papers reporting clinical studies should, where appropriate, contain a statement that they have been carried out with ethics committee approval. Papers disregarding the welfare of experimental animals will be rejected. Studies should be carried out in accordance with the relevant national and local guidelines.

The editorial office will rapidly review manuscripts in order that new findings may appear with minimum delay. The editorial office will return to authors within 3 weeks, whenever possible, all papers that are found to be of insufficient priority for further consideration. Papers of high interest will be sent out for external review. Authors will normally be notified of acceptance, rejection, or need for revision within 6 weeks of submission. Once a manuscript is accepted contributors will be provided with an electronic pdf proof, and corrections must be returned within three working days.

\section{Types of article}

Annals of Oncology publishes material in the form of editorials, original articles, letters, reviews, industry corner, and special articles.

Editorials. Editorials are solicited by the editor and are generally related to a paper published in the same issue. Length and format of the editorial will be agreed upon between editor and author.

Original articles. Full articles should generally be no longer than 4500 words, excluding manuscript heading, abstract, acknowledgements, and funding. Tables and figures are not limited in number but no more than 6 in total is recommended; each (including their legends, captions and footnotes) will count as 150 words towards the total word count of 4500; tables with excessive word counts will have the total words included in the final manuscript word count, however, extended material may be published as Supplementary Materials. References are unrestricted in number, therefore not counted in the total word count.

In the case of Supplementary Material, please indicate if it can be published online only. Online-only Supplementary Material should be uploaded in separate file(s), and described in the manuscript, in order to allow proper linking.

Figures, tables and references must be prepared according to specific instructions (see below). There is no limit on the number of figures or tables, but please consider that the journal is limited for space and that it may be possible to present some figures and tables as online only Supplementary Data. For further information see Supplementary material section below. Supplementary tables or figures should be named and numbered accordingly (S1, S2 etc.) in the manuscript and in the file.

Similarly, it may be possible to present an extended bibliography for online-only presentation. 
Plain Language Summaries are not required, but can be included as supplementary files for online publication only.

Pre-submission queries are welcome, and will be properly handled only if submitted via our online database. To do so, please access or create your Author profile at https://mc.manuscriptcentral.com/annonc.

Author Dashboard / Start New Submission

As this is a "partial submission" (and not a full manuscript submission), you are required to fill in the following fields only: Manuscript type (Review/Letter/Original Article/etc.) - Step 1 Abstract or a Short Description - Step 1 Abstract / Description file - or ms file if already available - Step 2 Three Keywords - Step 3 Corresponding Author Name (co-authors are not requested) - Step 4 Editor's Name - Step 5

Here you can add the editor's name, if you wish to address your presubmission to one of our editors (the e-mail address can be annals1@esmo.org)

Otherwise, to bypass this step, please add three fictitious names such as:

Ref1Name/Ref1Surname/Ref1@ref.org

Ref2Name/Ref2Surname/Ref2@ref.org

Ref3Name/Ref3Surname/Ref3@ref.org Manuscript Subtype (Breast/Thoracic Tum/etc.) - Step 6

All remaining fields can bypassed typing "N/A" or selecting any required "checkbox". Those fields will not be evaluated.

Letters to the editor. Letters to the editor are for correspondence relating to previously published articles, and only then within an appropriate time frame, or with interesting practice points, e.g. emerging side-effects of new drugs or rare diseases where there is a real practice issue.

Letters are welcome and will be published if appropriate. They should be no longer than 500 words and a maximum of five references; one table or figure is acceptable if absolutely necessary. No abstract is required.

Reviews. Meeting reports can only be considered for publication as reviews under exceptional circumstances; in such cases, the report should not simply be a report of new data presented but an attempt to synthesise the state of the art in a particular field.

Consensus documents based on the views of ad hoc expert panels are no longer acceptable, unless the panel was convened under the auspices of a widely recognised body or meeting and identified as such in the title.

The journal places no restriction on the style of review: narrative reviews, systematic reviews, and meta-analyses will all be considered.

Reviews are generally solicited by the editor. Unsolicited contributions will be considered although are rarely accepted. These should be submitted to the journal directly online for a rapid response.

Review manuscripts summarize the state-of-the-art in a particular field. Reviews should be no longer than 5000 words, on first submission, excluding manuscript heading, abstract, references (which are unrestricted in number, therefore not counted in the total word count), acknowledgements, funding, tables and figures. In the case of Supplementary Material, please indicate if it can be published online only; confidential materials for review only should be clearly indicated as such. If so, please upload it in separate file(s) (see appendices section). There is no limit on the number of figures or tables, but please consider that the journal is limited for space and that it may be possible to present some figures and tables as online only. Similarly, it may be possible to present an extended bibliography for online-only presentation.

\section{Word Counts}

Manuscripts that marginally exceed the stated word counts (not more than 10\%) will not be automatically rejected on the grounds of length alone, although immediate rejection remains a possibility, if the editors deem it necessary on the grounds of insufficient interest. If an overlong manuscript is submitted to peer review, shortening of the manuscript may be required if the manuscript is returned for revision. 
When providing word counts please indicate which word processing software and which version you are using.

\section{Page charges}

This journal has no page charges.

\section{BEFORE YOU BEGIN}

\section{Ethics in publishing}

Please see our information on Ethics in publishing.

\section{Studies in humans and animals}

If the work involves the use of human subjects, the author should ensure that the work described has been carried out in accordance with The Code of Ethics of the World Medical Association (Declaration of Helsinki) for experiments involving humans. The manuscript should be in line with the Recommendations for the Conduct, Reporting, Editing and Publication of Scholarly Work in Medical Journals and aim for the inclusion of representative human populations (sex, age and ethnicity) as per those recommendations. The terms sex and gender should be used correctly.

Authors should include a statement in the manuscript that informed consent was obtained for experimentation with human subjects. The privacy rights of human subjects must always be observed.

All animal experiments should comply with the ARRIVE guidelines and should be carried out in accordance with the U.K. Animals (Scientific Procedures) Act, 1986 and associated guidelines, EU Directive 2010/63/EU for animal experiments, or the National Research Council's Guide for the Care and Use of Laboratory Animals and the authors should clearly indicate in the manuscript that such guidelines have been followed. The sex of animals must be indicated, and where appropriate, the influence (or association) of sex on the results of the study.

\section{Informed consent and patient details}

Studies on patients or volunteers require ethics committee approval and informed consent, which should be documented in the paper. Appropriate consents, permissions and releases must be obtained where an author wishes to include case details or other personal information or images of patients and any other individuals in an Elsevier publication. Written consents must be retained by the author but copies should not be provided to the journal. Only if specifically requested by the journal in exceptional circumstances (for example if a legal issue arises) the author must provide copies of the consents or evidence that such consents have been obtained. For more information, please review the Elsevier Policy on the Use of Images or Personal Information of Patients or other Individuals. Unless you have written permission from the patient (or, where applicable, the next of kin), the personal details of any patient included in any part of the article and in any supplementary materials (including all illustrations and videos) must be removed before submission.

\section{Declaration of Potential Conflicts of Interest}

Any financial interests or connections, direct or indirect, or other situations that might raise the question of bias in the work reported or the conclusions, implications or opinions stated - including pertinent commercial or other sources of funding for the individual author(s) or for the associated department(s) or organization(s), personal relationships, or direct academic competition - should be disclosed. Examples of potential conflicts of interest include employment, consultancies, stock ownership, honoraria, paid expert testimony, patent applications/registrations, and grants or other funding.

If there are none, the disclosure should say: 'The authors have declared no conflicts of interest.'

If one or a few authors have a conflict to disclose, further to that statement, there should be an additional statement for those remaining authors who do not have any, e.g. 'All remaining authors have declared no conflicts of interest.'

\section{Declaration of generative AI in scientific writing}

The below guidance only refers to the writing process, and not to the use of AI tools to analyse and draw insights from data as part of the research process. 
Where authors use generative artificial intelligence (AI) and AI-assisted technologies in the writing process, authors should only use these technologies to improve readability and language. Applying the technology should be done with human oversight and control, and authors should carefully review and edit the result, as AI can generate authoritative-sounding output that can be incorrect, incomplete or biased. AI and AI-assisted technologies should not be listed as an author or co-author, or be cited as an author. Authorship implies responsibilities and tasks that can only be attributed to and performed by humans, as outlined in Elsevier's AI policy for authors.

Authors should disclose in their manuscript the use of AI and AI-assisted technologies in the writing process by following the instructions below. A statement will appear in the published work. Please note that authors are ultimately responsible and accountable for the contents of the work.

\section{Disclosure instructions}

Authors must disclose the use of generative AI and AI-assisted technologies in the writing process by adding a statement at the end of their manuscript in the core manuscript file, before the References list. The statement should be placed in a new section entitled 'Declaration of Generative AI and AIassisted technologies in the writing process'.

Statement: During the preparation of this work the author(s) used [NAME TOOL / SERVICE] in order to [REASON]. After using this tool/service, the author(s) reviewed and edited the content as needed and take(s) full responsibility for the content of the publication.

This declaration does not apply to the use of basic tools for checking grammar, spelling, references etc. If there is nothing to disclose, there is no need to add a statement.

\section{Submission declaration and verification}

Submission of an article implies that the work described has not been published previously (except in the form of an abstract, a published lecture or academic thesis, see 'Multiple, redundant or concurrent publication' for more information), that it is not under consideration for publication elsewhere, that its publication is approved by all authors and tacitly or explicitly by the responsible authorities where the work was carried out, and that, if accepted, it will not be published elsewhere in the same form, in English or in any other language, including electronically without the written consent of the copyrightholder. To verify compliance, your article may be checked by Crossref Similarity Check and other originality or duplicate checking software.

\section{Preprints}

Please note that preprints can be shared anywhere at any time, in line with Elsevier's sharing policy. Sharing your preprints e.g. on a preprint server will not count as prior publication (see 'Multiple, redundant or concurrent publication' for more information).

\section{Use of inclusive language}

Inclusive language acknowledges diversity, conveys respect to all people, is sensitive to differences, and promotes equal opportunities. Content should make no assumptions about the beliefs or commitments of any reader; contain nothing which might imply that one individual is superior to another on the grounds of age, gender, race, ethnicity, culture, sexual orientation, disability or health condition; and use inclusive language throughout. Authors should ensure that writing is free from bias, stereotypes, slang, reference to dominant culture and/or cultural assumptions. We advise to seek gender neutrality by using plural nouns ("clinicians, patients/clients") as default/wherever possible to avoid using "he, she," or "he/she." We recommend avoiding the use of descriptors that refer to personal attributes such as age, gender, race, ethnicity, culture, sexual orientation, disability or health condition unless they are relevant and valid. When coding terminology is used, we recommend to avoid offensive or exclusionary terms such as "master", "slave", "blacklist" and "whitelist". We suggest using alternatives that are more appropriate and (self-) explanatory such as "primary", "secondary", "blocklist" and "allowlist". These guidelines are meant as a point of reference to help identify appropriate language but are by no means exhaustive or definitive.

\section{Reporting sex- and gender-based analyses}

\section{Reporting guidance}

For research involving or pertaining to humans, animals or eukaryotic cells, investigators should integrate sex and gender-based analyses (SGBA) into their research design according to funder/ sponsor requirements and best practices within a field. Authors should address the sex and/or gender dimensions of their research in their article. In cases where they cannot, they should discuss this 
as a limitation to their research's generalizability. Importantly, authors should explicitly state what definitions of sex and/or gender they are applying to enhance the precision, rigor and reproducibility of their research and to avoid ambiguity or conflation of terms and the constructs to which they refer (see Definitions section below). Authors can refer to the Sex and Gender Equity in Research (SAGER) guidelines and the SAGER guidelines checklist. These offer systematic approaches to the use and editorial review of sex and gender information in study design, data analysis, outcome reporting and research interpretation - however, please note there is no single, universally agreed-upon set of guidelines for defining sex and gender.

\section{Definitions}

Sex generally refers to a set of biological attributes that are associated with physical and physiological features (e.g., chromosomal genotype, hormonal levels, internal and external anatomy). A binary sex categorization (male/female) is usually designated at birth ("sex assigned at birth"), most often based solely on the visible external anatomy of a newborn. Gender generally refers to socially constructed roles, behaviors, and identities of women, men and gender-diverse people that occur in a historical and cultural context and may vary across societies and over time. Gender influences how people view themselves and each other, how they behave and interact and how power is distributed in society. Sex and gender are often incorrectly portrayed as binary (female/male or woman/man) and unchanging whereas these constructs actually exist along a spectrum and include additional sex categorizations and gender identities such as people who are intersex/have differences of sex development (DSD) or identify as non-binary. Moreover, the terms "sex" and "gender" can be ambiguous-thus it is important for authors to define the manner in which they are used. In addition to this definition guidance and the SAGER guidelines, the resources on this page offer further insight around sex and gender in research studies.

\section{Changes to authorship}

Authors are expected to consider carefully the list and order of authors before submitting their manuscript and provide the definitive list of authors at the time of the original submission. Any addition, deletion or rearrangement of author names in the authorship list should be made only before the manuscript has been accepted and only if approved by the journal Editor. To request such a change, the Editor must receive the following from the corresponding author: (a) the reason for the change in author list and (b) written confirmation (e-mail, letter) from all authors that they agree with the addition, removal or rearrangement. In the case of addition or removal of authors, this includes confirmation from the author being added or removed.

Only in exceptional circumstances will the Editor consider the addition, deletion or rearrangement of authors after the manuscript has been accepted. While the Editor considers the request, publication of the manuscript will be suspended. If the manuscript has already been published in an online issue, any requests approved by the Editor will result in a corrigendum.

\section{Reporting Clinical Trials}

Authors reporting clinical trials may find the guidelines given in the report of Simon and Wittes useful. (Simon R, Wittes RE. Methodologic guidelines for reports of clinical trials. Cancer Treat Rep 1985; 69: 1-3.) Particularly critical is the correct application and presentation of survival analyses: useful guidelines can be found in the appendix of the report by D. G. Altman et al. (Altman DG, De Stavola $\mathrm{BL}$, Love SB, Stepniewska KA. Review of survival analyses published in cancer journals. Br J Cancer 1995; 72: 511-518).

The quality of data reporting on randomized clinical trials will be evaluated following the rules and checklist of the CONSORT statement (CONSORT 2010 Statement: Updated Guidelines for Reporting Parallel Group Randomized Trials. Schulz KF, Altman DG, Moher D et al. Ann Intern Med 2010; 152: 1-7); if required, material concerning this statement will be forwarded to the authors (the CONSORT Flow Diagarm is available here: http://www.consort-statement.org/consort-statement/ flow-diagram). Randomized clinical trials, which have begun after 1 January 1997, must be monitored and carried out in a manner permitting an absolute adherence to the rules of the CONSORT statement, as regards publication of their results. Potentially acceptable manuscripts will be submitted for statistical review. Any registered clinical trial number should be indicated after the abstract.

\section{Phase I trials}


Reports of phase I studies can only be considered where there are additional translational research components. In exceptional cases, specifically where a remarkable response rate was observed, translational research is not required. The reporting of response rates for rare tumors is in any case encouraged.

\section{Phase II trials}

Reports of phase II studies should be testing novel and innovative ideas and producing data that form the basis for important RCTs, or data that clearly suggest the lack of potential for such RCTs. There is no objection to negative phase II studies, provided they give clear guidance for future work. Single-arm phase II studies with combination schedules that include established drugs, but without additional translational research, cannot be considered. Phase II studies should use recognised statistical designs.

\section{Phase III trials}

Submission of reports of prospective, randomised phase III studies is encouraged. Fast-track facilities for editorial handling and, potentially, publication (to print) are available subject to agreement via a pre-submission query. Please contact the Editorial office.

Longer-term follow up reports of previously reported phase III trials are welcomed.

Studies of prognostic markers of no real future clinical utility and single biomarkers studies cannot be considered. These studies should be prospective and have a clear view of the practical clinical applications of the results. Retrospective analysis of biomarkers will be considered if done within the framework of data collected from a prospective trial, with appropriate statistics and with multivariate analysis that includes established predictive/prognostic markers.

Reports of tumor registry studies need to have clear clinical relevance; pre- submission queries are encouraged.

The journal is committed to translational research for the development of oncology, including basic, i.e. wholly preclinical, cancer research where clinical potential is clear.

\section{Copyright}

Upon acceptance of an article, authors will be asked to complete a 'Journal Publishing Agreement' (see more information on this). An e-mail will be sent to the corresponding author confirming receipt of the manuscript together with a 'Journal Publishing Agreement' form or a link to the online version of this agreement.

Subscribers may reproduce tables of contents or prepare lists of articles including abstracts for internal circulation within their institutions. Permission of the Publisher is required for resale or distribution outside the institution and for all other derivative works, including compilations and translations. If excerpts from other copyrighted works are included, the author(s) must obtain written permission from the copyright owners and credit the source(s) in the article. Elsevier has preprinted forms for use by authors in these cases.

\section{Author rights}

As an author you (or your employer or institution) have certain rights to reuse your work. More information.

\section{Disclailmer}

Practitioners and researchers must always rely on their own experience and knowledge in evaluating and using any information, methods, compounds or experiments described herein. Because of rapid advances in the medical sciences, in particular, independent verification of diagnoses and drug dosages should be made. To the fullest extent of the law, no responsibility is assumed by the publisher, the European Society for Medical Oncology and Japanese Society for Medical Oncology for any injury and/or damage to persons or property as a matter of products liability, negligence or otherwise, or from any use or operation of any methods, products, instructions or ideas contained in the material herein. 
Details of all funding sources for the work in question are mandatory; please include a funding statement before the disclosure, or add 'none declared'.

The following rules should be followed.

The sentence should begin: 'This work was supported by ?' The full official funding agency name should be given, i.e. 'the National Cancer Institute at the National Institutes of Health' or simply 'National Institutes of Health' not 'NCI' (one of the 27 sub-institutions) or 'NCI at NIH' (full RINapproved list of UK funding agencies). Grant numbers should be given in brackets as follows: '[grant number $x x x x$ ]' Multiple grant numbers should be separated by a comma as follows: '[grant numbers XXXX, yyyy]' Agencies should be separated by a semi-colon (plus 'and' before the last funding agency) or grant number 'to [author initials]'. Where individuals need to be specified for certain sources of funding the following text should be added after the relevant agency or grant number 'to [author initials]'. Please state if no grant number is applicable

An example is given here: 'This work was supported by the National Institutes of Health [AA123456 to C.S., BB765432 to M.H.]; and the Alcohol \& Education Research Council [hfygr667789].'

The NIH public access policy requires authors to submit accepted manuscripts that arise from NIH funding to PubMed Central, immediately upon acceptance for publication, to be made publicly available after 12 months. Elsevier and NIH have an arrangement that supports NIH-funded authors and NIH employees who publish with Elsevier to comply with NIH's public access requirements.

Crossref Funding Data Registry: In order to meet your funding requirements authors are required to name their funding sources, or state if there are none, during the submission process. For further information on this process or to find out more about the CHORUS initiative please see: https:// www.elsevier.com/en-gb/about/open-science/open-access/chorus

\section{Open access}

Please visit our Open Access page for more information.

Language (usage and editing services)

Please write your text in good English (American or British usage is accepted, but not a mixture of these). Authors who feel their English language manuscript may require editing to eliminate possible grammatical or spelling errors and to conform to correct scientific English may wish to use the English Language Editing service available from Elsevier's Author Services.

\section{Submit your artïcle}

Please submit your article via the online submission web site.

\section{PREPARATION}

Use of word processing software

It is important that the file be saved in the native format of the word processor used. The text should be in single-column format. Keep the layout of the text as simple as possible. Most formatting codes will be removed and replaced on processing the article. In particular, do not use the word processor's options to justify text or to hyphenate words. However, do use bold face, italics, subscripts, superscripts etc. When preparing tables, if you are using a table grid, use only one grid for each individual table and not a grid for each row. If no grid is used, use tabs, not spaces, to align columns. The electronic text should be prepared in a way very similar to that of conventional manuscripts (see also the Guide to Publishing with Elsevier). Note that source files of figures, tables and text graphics will be required whether or not you embed your figures in the text. See also the section on Electronic artwork.

To avoid unnecessary errors you are strongly advised to use the 'spell-check' and 'grammar-check' functions of your word processor.

\section{Article Structure}

Divide your article into clearly defined sections. Each subsection is given a brief heading. Each heading should appear on its own separate line. Subsections should be used as much as possible when crossreferencing text: refer to the subsection by heading as opposed to simply 'the text'. 
Generally, an original article should be structured as follows: introduction; methods-patients and methods-materials and methods-etc; results; discussion (conclusion may be used as a subheading in the discussion); acknowledgements; funding; disclosure; references.

\section{Appendices}

If there is more than one appendix, they should be identified as $A, B$, etc. Formulae and equations in appendices should be given separate numbering: Eq. (A.1), Eq. (A.2), etc.; in a subsequent appendix, Eq. (B.1) and so on. Similarly for tables and figures: Table A.1; Fig. A.1, etc.

\section{Essential title page information}

Number the pages consecutively with the first page containing the following headings:

article type title author(s) list: first name(s) written with initials only, and followed by the last name - e.g. J. E. Smith

There is no restriction on the number of authors; manuscripts can have as many authors as needed affiliation(s) list: the affiliation list should be written as follows: Department/Division Name (in English), Affiliation/Institution, City, Country Manuscript social media information: all available social media handles you would like your manuscript to be linked, e.g. Twitter, Facebook, Instagram, url of websites, etc full address for correspondence Corresponding Author should be designated. For Original Article and Review:

this should be written as follows: title of corresponding author (Mr/Mrs/Ms/Dr/Prof ) without academic title (MD, PhD, etc.), author name (written with first name, middle initial, then last name) Department/ Division/Unit Name (in English), Affiliation/Institution, street address, city, postal code, country, country code and telephone number, email address For Editorial and Letter to the Editor:

Corresponding Author e-mail address in brackets, e.g. (*E-mail:???.@?..) Author ORCID indemnifiers; for further information on ORCID see https://www.elsevier.com/connect/authors-update/tenreasons-to-get-and-use-an-orcid-id!

\section{Structured abstract}

Please provide a short summary of 300 words or less. The summary should not contain any undefined abbreviations or unspecified references. Summaries should be organized and formatted according to the following headings: (1) Background, (2) Patients and methods, (3) Results and (4) Conclusion(s). Authors may substitute 'Design' or 'Materials and methods' for 'Patients and methods' in summaries of Review articles or of papers dealing with basic research.

\section{Keywords}

Immediately after the abstract, provide a maximum of 6 keywords, using American spelling and avoiding general and plural terms and multiple concepts (avoid, for example, 'and', 'of'). Be sparing with abbreviations: only abbreviations firmly established in the field may be eligible. These keywords will be used for indexing purposes.

\section{Acknowledgements}

Collate acknowledgements in a separate section at the end of the article before the Funding and do not, therefore, include them on the title page, as a footnote to the title or otherwise. List here those individuals who provided help during the research (e.g., providing language help, writing assistance or proof reading the article, etc.).

\section{Decimal numerals}

To enhance readability and clarity of the text as well as tables and figures, decimal numerals should - with the obvious exception of $P$-values - be rounded to the unit whenever possible (i.e. in all cases in which the rounding procedure does not change the meaning). Value " $N$ " and " $P$ " should always be written in italic.

\section{Highlights}

Highlights are a short collection of bullet points that convey the core findings and provide readers with a quick textual overview of the article. These three to five bullet points describe the essence of the research (e.g. results or conclusions) and highlight what is distinctive about it. Highlights will be displayed in online search result lists, the contents list and in the online article, but will not (yet) appear in the article PDF file. Note that highlights are only required for Original Articles and Reviews.

Specifications: Include 3 to 5 highlights. There should be a maximum of 125 characters, including spaces, per highlight. Only the core results of the paper should be covered. 
Collaborators (sometimes called non-author contributors) will be listed on PubMed as collaborators rather than authors. In order to be indexed as collaborators, the names of the consortium or working group members should be listed in an Appendix in the main text document, not as supplementary material. The consortium or working group should also be included in the main author list. PubMed will list the names of individual group members who are authors or collaborators. There should be a note associated with the author list clearly stating that the individual names are elsewhere in the paper and whether those names are authors or collaborators. Collaborator names are searchable on PubMed in the same way as authors.

Artwork

Electronic artwork

General points

- Make sure you use uniform lettering and sizing of your original artwork.

- Embed the used fonts if the application provides that option.

- Aim to use the following fonts in your illustrations: Arial, Courier, Times New Roman, Symbol, or use fonts that look similar.

- Number the illustrations according to their sequence in the text.

- Use a logical naming convention for your artwork files.

- Provide captions to illustrations separately.

- Size the illustrations close to the desired dimensions of the published version.

- Submit each illustration as a separate file.

- Ensure that color images are accessible to all, including those with impaired color vision.

A detailed guide on electronic artwork is available.

You are urged to visit this site; some excerpts from the detailed information are given here.

Formats

Figures in Annals of Oncology are recoloured according to the style of the journal, therefore all figures submitted should be provided in an editable format. Please download this guideline.

\section{Please do not:}

- Supply files that are optimized for screen use (e.g., GIF, BMP, PICT, WPG); these typically have a low number of pixels and limited set of colors:

- Supply files that are too low in resolution;

- Submit graphics that are disproportionately large for the content.

Color artwork

Please make sure that artwork files are in an acceptable format. If, together with your accepted article, you submit usable color figures then Elsevier will ensure, at no additional charge, that these figures will appear in color online (e.g., ScienceDirect and other sites) regardless of whether or not these illustrations are reproduced in color in the printed version. Further information on the preparation of electronic artwork.

\section{Cover Images}

Authors are particularly encouraged to submit colour images relating to their article, but not necessarily part of it, for consideration as a potential cover image for a journal issue. Files should be uploaded separately and labelled as potential cover art; the images should also be mentioned in the submission letter.

Permissions

As a general rule, permission should be sought from the rightsholder to reproduce any substantial part of a copyrighted work. This includes any text, illustrations, charts, tables, photographs, or other material from previously published sources.

To read our full Permissions Guidelines and determine if you require permission, please click here.

Figure captions

Ensure that each illustration has a caption. Supply captions separately, not attached to the figure. A caption should comprise a brief title (not on the figure itself) and a description of the illustration. Keep text in the illustrations themselves to a minimum but explain all symbols and abbreviations used. 
Please submit tables as editable text and not as images. Tables can be placed either next to the relevant text in the article, or on separate page(s) at the end. Number tables consecutively in accordance with their appearance in the text and place any table notes below the table body. Be sparing in the use of tables and ensure that the data presented in them do not duplicate results described elsewhere in the article. Please avoid using vertical rules and shading in table cells. Value " N " and " P " should always be written in italic, throughout tables, figures and manuscript.

\section{Web references}

As a minimum, the full URL should be given and the date when the reference was last accessed. Any further information, if known (DOI, author names, dates, reference to a source publication, etc.), should also be given. Web references can be listed separately (e.g., after the reference list) under a different heading if desired, or can be included in the reference list.

\section{Preprint references}

Where a preprint has subsequently become available as a peer-reviewed publication, the formal publication should be used as the reference. If there are preprints that are central to your work or that cover crucial developments in the topic, but are not yet formally published, these may be referenced. Preprints should be clearly marked as such, for example by including the word preprint, or the name of the preprint server, as part of the reference. The preprint DOI should also be provided.

\section{References}

Please ensure that every reference cited in the text is also present in the reference list (and vice versa). Any references cited in the abstract must be given in full. Unpublished results and personal communications are not recommended in the reference list, but may be mentioned in the text. If these references are included in the reference list they should follow the standard reference style of the journal and should include a substitution of the publication date with either 'Unpublished results' or 'Personal communication'. Citation of a reference as 'in press' implies that the item has been accepted for publication and a copy of the title page of the relevant article must be submitted.

In the text, a reference identified by means of an author's name should be followed by the reference number in superscripts. When there are more than two authors, only the first author's name should be mentioned, followed by et al.

\section{Examples.}

Winograd $^{1}$

Bullen and Bennett ${ }^{2}$

Wilson et al. ${ }^{3}$

References to books, journal articles, articles in collections and conference or workshop proceedings, and technical reports should be listed at the end of the article in numbered order following the Index Medicus style (see examples below). References to abstracts and Letters to the editor must be identified as such. List all authors if three or fewer; if four or more, list only the first three, followed by et al. Articles in preparation or articles submitted for publication, unpublished observations, personal communications, etc. should not be included in the reference list but should only be mentioned in the article text (e.g. T. Moore, personal communication).

References to books should include the author's name; year of publication; title; page numbers where appropriate; publisher; place of publication, in the order given in the example below.

Pecorino L. Molecular Biology of Cancer Mechanisms, Targets, and Therapeutics, 3rd edition: Oxford University Press, Oxford, 2012.

References to articles in an edited collection should include the author's name; year of publication; article title; editor's name; title of collection; first and last page numbers; publisher; place of publication, in the order given in the example below.

Brennan MF, Alektiar KM, Maki RG. Soft tissue sarcoma. In DeVita VT Jr, Hellman S, Rosenberg SA (eds): Cancer: Principles and Practice of Oncology, 6th edition. Philadelphia, PA: Lippincott Williams \& Wilkins 2001; 1841-1980. 
References to articles in periodicals should include the author's name; year of publication; article title; full abbreviated title of periodical; volume number (issue number where appropriate); first and last page numbers, in the order given in the example below.

Schrodi S, Braun M, Andrulat A et al. Outcome of breast cancer patients with low hormone receptor positivity:analysis of a 15-year population-based cohort. Ann Oncol 2021; 32: 1410-1424.

If an article has been published online but has not yet been given issue or page numbers please use the Digital Object Identifier (doi) number when referencing the article as in the example below.

Dimitriou F, Lo SN, Tan AC et al. FDG-PET to predict long-term outcome from anti-PD-1 therapy in metastatic melanoma. Ann Oncol 2021. October 20 [Epub ahead of print] doi: 10.1016/ j.annonc.2021.10.003

\section{Supplementary material}

Supplementary material such as applications, images and sound clips, can be published with your article to enhance it. Submitted supplementary items are published exactly as they are received (Excel or PowerPoint files will appear as such online). Please submit your material together with the article and supply a concise, descriptive caption for each supplementary file. If you wish to make changes to supplementary material during any stage of the process, please make sure to provide an updated file. Do not annotate any corrections on a previous version. Please switch off the 'Track Changes' option in Microsoft Office files as these will appear in the published version.

\section{Graphical Abstracts}

Graphical abstracts may be presented to summarize the contents of the article in a concise, pictorial form designed to capture the attention of a wide readership. Graphical abstracts should be submitted as a separate supplementary file in the online submission system. Image size: Please provide an image with a minimum of $531 \times 1328$ pixels ( $\mathrm{h} X \mathrm{w}$ ) or proportionally more. The image should be readable at a size of $5 \times 13 \mathrm{~cm}$ using a regular screen resolution of $96 \mathrm{dpi}$. Preferred file types: TIFF, EPS, PDF or MS Office files.

The journal also accepts video material and animation sequences as supplementary material. All submitted files should be properly labelled so that they directly relate to the video file's content. In order to ensure that your video or animation material is directly usable, please provide the file in one of our recommended file formats with a preferred maximum size of 150 MB per file, 1 GB in total. For more detailed instructions please visit our instruction pages.

\section{AFTER ACCEPTANCE}

\section{Availability of accepted article}

This journal makes articles available online as soon as possible after acceptance. This concerns the Journal Pre-proofs (both in HTML and PDF format), which have undergone enhancements after acceptance, such as the addition of a cover page and metadata, and formatting for readability, but are not yet the definitive versions of record. A Digital Object Identifier (DOI) is allocated, thereby making it fully citable and searchable by title, author name(s) and the full text. The article's PDF also carries a disclaimer stating that it is an unedited article. Subsequent production stages will simply replace this version.

\section{Proofs}

One set of page proofs (as PDF files) will be sent by e-mail to the corresponding author (if we do not have an e-mail address then paper proofs will be sent by post) or a link will be provided in the email so that authors can download the files themselves. To ensure a fast publication process of the article, we kindly ask authors to provide us with their proof corrections within two days. Elsevier now provides authors with PDF proofs which can be annotated; for this you will need to download the free Adobe Reader, version 9 (or higher). Instructions on how to annotate PDF files will accompany the proofs (also given online). The exact system requirements are given at the Adobe site.

If you do not wish to use the PDF annotations function, you may list the corrections (including replies to the Query Form) and return them to Elsevier in an e-mail. Please list your corrections quoting line number. If, for any reason, this is not possible, then mark the corrections and any other comments (including replies to the Query Form) on a printout of your proof and scan the pages and return via email. Please use this proof only for checking the typesetting, editing, completeness and correctness of the text, tables and figures. Significant changes to the article as accepted for publication will only be considered at this stage with permission from the Editor. We will do everything possible to get your 
article published quickly and accurately. It is important to ensure that all corrections are sent back to us in one communication: please check carefully before replying, as inclusion of any subsequent corrections cannot be guaranteed. Proofreading is solely your responsibility.

\section{Offprints}

The corresponding author will, at no cost, receive a customized Share Link providing 50 days free access to the final published version of the article on ScienceDirect. The Share Link can be used for sharing the article via any communication channel, including email and social media. For an extra charge, paper offprints can be ordered via the offprint order form which is sent once the article is accepted for publication. Corresponding authors who have published their article gold open access do not receive a Share Link as their final published version of the article is available open access on ScienceDirect and can be shared through the article DOI link.

\section{AUTHOR INQUIRIES}

Visit the Elsevier Support Center to find the answers you need. Here you will find everything from Frequently Asked Questions to ways to get in touch.

You can also check the status of your submitted article or find out when your accepted article will be published.

\section{Requests about the status of your manuscript}

Once a manuscript is submitted, you can follow manuscript developments from your online account, at the Annals of Oncology online submission website http://mc.manuscriptcentral.com/annonc. In particular questions about the status of your manuscript should be addressed to the Editorial Office at annals1@esmo.org or annals2@esmo.org. Please include your manuscript number (this number was sent to you upon receipt of your manuscript). However, considering the processes are confidential, the Editorial Office can rarely provide more details than those available in the author account; queries not from the author will not be answered.

Additional information can be obtained from:

\section{Annals of Oncology - Editorial Office}

Annals of Oncology

Via Ginevra 4

$\mathrm{CH}-6900$ Lugano

Switzerland

\section{Telephone:}

+41 (0)91-973-1908/10 